\title{
Efficient Numerical Modeling of Field Diffusion in High-Temperature Superconducting Wires
}

\author{
Igor O. Golosnoy and Jan K. Sykulski, Fellow, IEEE \\ School of Electronics and Computer Science, University of Southampton, Southampton SO17 1BJ, U.K.
}

\begin{abstract}
Application of a finite-volume front-fixing method to various front-type problems with moving boundaries and nonlinear material properties is extended to two-dimensional (2D) problems. Attention needs to be paid to conservation properties of the algorithm and accurate solutions close to the moving boundaries. Advantages of the method are highlighted, and particular implementation difficulties discussed. The algorithm has been tested against analytical solutions of diffusion problems possessing cylindrical symmetry.
\end{abstract}

Index Terms-Coupled problems, front-fixing method, moving boundary problems.

\section{INTRODUCTION}

$\mathbf{U}$ SING high-temperature superconductors (HTS) in modern devices promises significant energy savings, but also exposes difficulties in dealing with short-circuit faults or other impulse loads. Such intensive impacts require coupled treatment of both electromagnetic and thermal parts of the problem since material properties of HTS are quite sensitive to temperature [1]. Standard numerical modeling of the pulse events on fixed grids provides valuable assistance in the equipment design, but existing computational techniques often fail to achieve appropriate balance between accuracy and efficiency. More complex methods - such as adaptive meshes, front fixing, and level sets methods [2] —offer advantages in applications to impulse problems, but need to be assessed and probably adapted for the particular problem. The paper uses analytical solutions of common front-type problems for the evaluation of the numerical methods. The main emphasis is on the analysis of the front-fixing technique [2] since it requires only a small adjustment of the computational algorithm in comparison with formulations using fixed grids [3]. Special attention is given to conservation properties of the algorithm and accurate solutions close to the moving boundaries.

\section{Formulation of the Problem}

The flow of an electric current in HTS can be formulated in terms of either magnetic or electric field diffusion [4], [5]. The electric field formulation is preferred for highly nonlinear processes since it provides much more stable solutions [5]. For this case, the governing equation takes the diffusion-like form

$$
\operatorname{curl}(\operatorname{curl} \mathbf{E})=-\mu_{0} \frac{\partial \mathbf{J}}{\partial t}
$$

in terms of the electric field $\mathbf{E}$ and current density $\mathbf{J}$, but has to be supplemented by a relationship between field $E$ and current density $J$. A strong flux creep $E-J$ characteristic is a specific feature of HTS materials. This is often described by the power law $E_{c}^{-1} E=\left(J_{c}^{-1} J\right)^{\alpha}$ [4], where the critical current

Manuscript received December 08, 2009; accepted February 13, 2010. Current version published July 21, 2010. Corresponding author: I. O. Golosnoy (e-mail: ig@ecs.soton.ac.uk).

Color versions of one or more of the figures in this paper are available online at http://ieeexplore.ieee.org.

Digital Object Identifier 10.1109/TMAG.2010.2043718 density $J_{c} \approx 10^{9} \mathrm{Am}^{-2}$ corresponds to a critical electric field $E_{c} \approx 10^{4} \mathrm{Vm}^{-1}$ and a large power exponent $\alpha$, which for practical materials could be about 20. Substitution of the material properties into (1) results in a formulation of the problem in terms of the electric field only. It should be noted that (1) can be rewritten in terms of the electric current only

$$
\operatorname{curl}\left(\operatorname{curl}\left[\left(J_{c}^{-1} J\right)^{\alpha} E_{c} \hat{\mathbf{J}}\right]\right)=-\mu_{0} \frac{\partial \mathbf{J}}{\partial t}
$$

but more commonly will be in terms of the electric field

$$
\operatorname{curl}(\operatorname{curl} \mathbf{E})=-\mu_{0} \frac{\partial\left[J_{c}\left(E_{c}^{-1} E\right)^{1 / \alpha} \hat{\mathbf{E}}\right]}{\partial t} .
$$

Both (2) and (3) have advantages and disadvantages. The time derivative in (2) does not include the nonlinear term, and this makes an implementation of conservation laws easier. On the other hand, absolute variation in the nonlinear term is smaller for (3) due to the $1 / \alpha$ power. For this reason, (3) is less sensitive to rounding errors in calculations of impulse events.

It is helpful to conduct tests in at least two-dimensional (2D) geometry. The existence of an analytical solution in cylindrical coordinates provides an opportunity to evaluate the technique's performance on curved boundaries using the Cartesian coordinate system. Consider a HTS wire with a circular cross section of radius $R$ with embedded electrically insulated very fine conductor in the center. If the current pulse $J_{z}(r, t)=I_{0} \delta(\hat{\mathbf{r}}) \delta(t-$ $\left.t_{0}\right)$ is applied to the conductor along the $\mathrm{z}$-axis at instant $t=t_{0}$, the dimensionless solution for (1) in the case of cylindrical symmetry can be derived as shown in [6]

$$
e(\rho, \tau)=\frac{1}{(\alpha \tau)}\left[\left(\frac{i_{0}}{4}\right)^{\frac{\alpha-1}{\alpha}}-\frac{\rho^{2}(\alpha-1)}{4 \alpha(\alpha \tau)^{1 / \alpha}}\right]^{\alpha /(\alpha-1)}
$$

with $e=i^{\alpha}$ and dimensionless length, time, electric field, and current defined as

$$
\begin{aligned}
\rho=\frac{r}{R}, \quad \tau= & \frac{\left(t-t_{0}\right) E_{c}}{\mu_{0} J_{c} R^{2}}, \quad i_{0}=\frac{I_{0}}{J_{c} \pi R^{2}} \\
& \quad(\rho, \tau)=\frac{E_{z}(\rho, \tau)}{E_{c}}, \quad i(\rho, \tau)=\frac{J_{z}(\rho, \tau)}{J_{c}} .
\end{aligned}
$$

The equation is valid only inside $\rho \leq \rho_{b}(\tau)$ and during a limited time when $\rho_{b}(\tau) \leq 1$, where

$$
\rho_{b}(\tau)=\left[\frac{4 \alpha(\alpha \tau)^{1 / \alpha}}{(\alpha-1)}\left(\frac{i_{0}}{4}\right)^{\frac{\alpha-1}{\alpha}}\right]^{1 / 2} .
$$




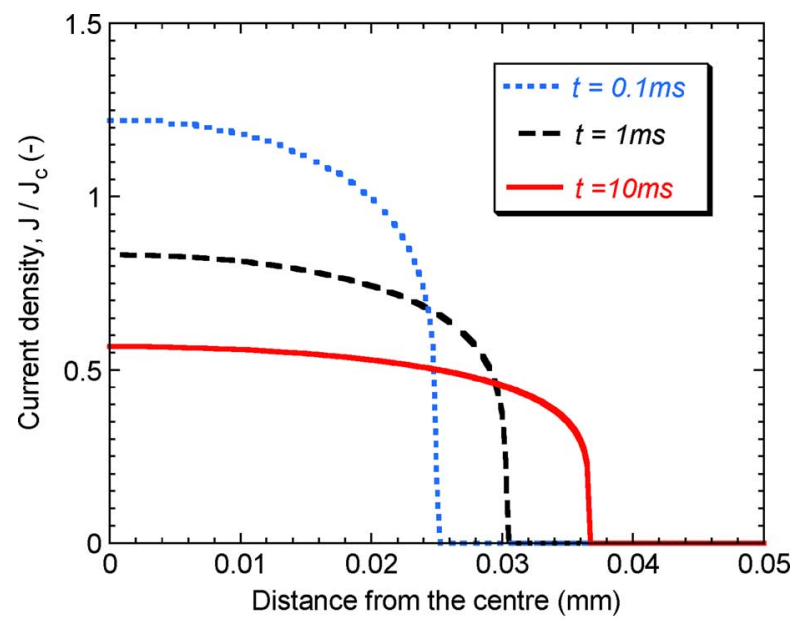

Fig. 1. Evolution of current density $J_{z}(r, t)$ in a HTS circular wire. The analytical prediction of electric field and current density for a wire with $I_{0}=2 \mathrm{~A}$, $R=0.5 \mathrm{~mm}$, and $\alpha=6$ is shown at different instances of time.

The field and the current are zero outside the front region. The electric field and the current gradually spread from the center of the wire toward the edges, and there is a sharp interface between the region with nonzero field and the outside part of the wire. The current density profiles at different instances of time are shown in Fig. 1.

Keeping in mind that the only nonzero component of electric field is in the $z$-direction, (3) may be written for the first quarter in Cartesian coordinates by taking account of notations (5) and the symmetry of the problem

$$
\begin{aligned}
\frac{\partial}{\partial \tau}\left(\text { signe } \cdot|e|^{1 / \alpha}\right) & =\frac{\partial^{2} e}{\partial x^{2}}+\frac{\partial^{2} e}{\partial y^{2}} \\
\rho^{2} & =x^{2}+y^{2}, \quad x, y \in[0,1] .
\end{aligned}
$$

Appropriate boundary conditions for (7) would be

$$
\left.\frac{\partial e}{\partial x}\right|_{y=0}=0,\left.\quad \frac{\partial e}{\partial y}\right|_{x=0}=0
$$

at the symmetry lines. Direct integration of (4) yields a conservation integral

$$
\left(\int_{0}^{1} \int_{0}^{1} i(x, y, \tau) d x d y\right)=\frac{\pi i_{0}}{4} \equiv \text { const. }
$$

By applying Ampere's law at any contour outside the boundary $\rho_{\mathrm{b}}$, we can conclude that

$$
\frac{\partial e}{\partial \hat{\mathbf{n}}}=0, \quad e=0
$$

at any point behind the front edge $\rho_{\mathrm{b}}$, with $\hat{\mathbf{n}}$ being the normal to the computational region boundary.

\section{Computational TechniQue}

\section{A. Front-Fixing Method}

Let us fix an extent of the computational region by some boundary $s(x, y)=0$ as described in [2]. In general, the transformed version of (7) contains mixed derivatives; this is not desirable for modeling of diffusion processes with sharp gradients since small time steps are required to suppress nonmonotonic oscillations in the numerical solution (see [7] for general theory of monotonic solutions). Fortunately, the boundary condition (10) is an implicit one and may be imposed at any point behind the real boundary. It is therefore convenient to consider a rectangular region with moving edges $x_{s}(\tau), y_{s}(\tau)$. An introduction of new variables $u=x / x_{s}, v=y / y_{s}$ fixes the extent of the computational domain to $0 \leq u, v \leq 1$ and results in

$$
\begin{aligned}
\frac{\partial p}{\partial \tau}= & \frac{1}{x_{s}}\left(\frac{d x_{s}}{d \tau} \frac{\partial}{\partial u}(u p)+y_{s} \frac{\partial^{2} e}{\partial u^{2}}\right) \\
& +\frac{1}{y_{s}}\left(\frac{d y_{s}}{d \tau} \frac{\partial}{\partial v}(v p)+x_{s} \frac{\partial^{2} e}{\partial v^{2}}\right), \quad \text { with } p=y_{s} x_{s} i .
\end{aligned}
$$

An introduction of $p$ in (11) is due to the fact that the integral of $p$ is actually conserved during the coordinate transformation and field diffusion. Additional equations to predict variations of $x_{s}, y_{s}$ with time should be added to (11) as discussed in the implementation section.

In order to derive a finite volume scheme, we have to integrate (11) over time and space intervals [8]. The space is discretized at $M+1$ points for $u$ and at $K+1$ points for $v$. The discretization points are defined by a fixed discretization of $u, v$, and they may be written as $u_{0}=0, u_{1}, \ldots, u_{M}=1, v_{0}=0, v_{1}, \ldots, v_{K}=1$. The finite volume discretization [8] of (11) is based on integration around the nodes and is fairly straightforward [9], [10]. The boundary conditions (8) and (10) appear naturally in

$$
\begin{aligned}
& \left.\frac{\partial e}{\partial u}\right|_{v=0}=0,\left.\quad \frac{\partial e}{\partial v}\right|_{u=0}=0 \\
& \left.\frac{\partial e}{\partial u}\right|_{v=1}=0,\left.\quad p\right|_{v=1}=0,\left.\quad \frac{\partial e}{\partial v}\right|_{u=1}=0,\left.\quad p\right|_{u=1}=0 .
\end{aligned}
$$

The finite volume discretization for (12) and (13) immediately follows from the general form of the finite volume integral.

\section{B. Numerical Scheme}

To develop unconditionally monotonic scheme, we restrict the consideration by combining an explicit method with an appropriate limiter [7], [8] for the convective part in (11) with a fully implicit method for diffusion fluxes. The integrals over time in (11) result in an operator finite volume form with the convective fluxes split in the $u$ and $v$ directions

$$
U\left(p^{n+1}-p^{n}\right)=L A_{u} p^{n}+L A_{v} p^{n}+D_{u} e^{n+1}+D_{v} e^{n+1}
$$

where $A$ and $D$ are $u$-, $v$-directional convection and diffusion operators, respectively, $U$ is a unity operator, and $L$ is a limiter [7], [8]. Standard centered spatial discretization is applied for $A$ and $D$ [8]. The limiter $L$ is chosen to be a linear combination of standard upwind/downwind with high-resolution schemes [7]. Tests on one-dimensional (1D) cylindrically symmetric problems [7], [8] indicate that appropriate choice of $L$ could provide second order accuracy in space.

\section{Fractional Steps}

The numerical solution of (14) could be optimized since: 1) the application of $A_{u}$ and $A_{v}$ together results in strict stability conditions; 2) relation between $p$ and $e$ is nonlinear; moreover, $D_{u}, D_{v}$ have broad spectra, and the choice of iteration parameters for standard methods, e.g. Gauss-Seidel, is not obvious. Both complications can be overcome by applying the so-called 

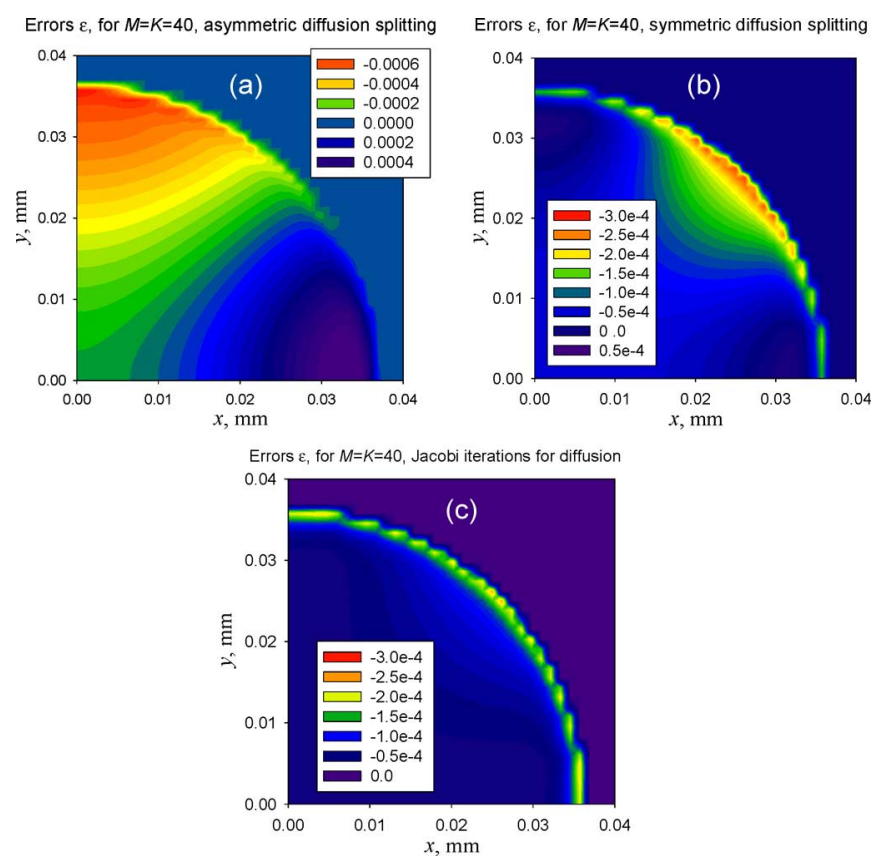

Fig. 2. Errors in predictions of electric field inside a HTS wire with $I_{0}=2 \mathrm{~A}$, $R=0.5 \mathrm{~mm}$, and $\alpha=6$ after $t=10 \mathrm{~ms}$. A constant time step $\delta_{\tau}=10^{-6} \mathrm{~s}$ and a grid with $M=K=40$ were used for predictions. (a) Asymmetric form of diffusion split (17) and (18) distorts the front shape: It becomes an ellipsoid extended in $x$-direction. (b) Symmetrization decreases the errors and shifts them to $x=y$ plane. (c) Coupled $u-v$ scheme (19) with Jacobi iterations produces minimal errors, but is very demanding in terms of computational time.

"fractional step" method, which replaces a sum on the left-hand side of (14) by a linked chain of $1 \mathrm{D}$ equations

$$
\begin{aligned}
U\left(p^{n+0.25}-p^{n}\right) & =L A_{u} p^{n} \\
U\left(p^{n+0.5}-p^{n+0.25}\right) & =L A_{v} p^{n+0.25} \\
U\left(p^{n+0.75}-p^{n+0.5}\right) & =D_{u} e^{n+0.75} \\
U\left(p^{n+1}-p^{n+0.75}\right) & =D_{v} e^{n+1} .
\end{aligned}
$$

It was found that strong nonlinearity of the diffusion operator introduces large errors (see Fig. 2), which can be significantly reduced by introducing a symmetric sequence: The orders of diffusion operators $D_{v}, D_{u}$ are swapped in (17) and (18) for the additional estimate of $p^{n+1}$, and the final value is obtained as a half sum of both estimates.

The solution of the equations has been described in [3]. The proposed method (15)-(18) requires of the order of $O(M K)$ operations and is therefore quite efficient. Another possible approach is to use Jacobi iterations [7] to solve

$$
U\left(p^{n+1}-p^{n+0.5}\right)=D_{u} e^{n+1}+D_{v} e^{n+1}
$$

linked with (15) and (16). Equation (19) should be solved simultaneously in both $u$ - and $v$-directions. The symmetry is preserved by (19), but Jacobi iterations require $O\left(M^{2} K^{2}\right)$ operations and are inefficient for fine grids.

\section{Implementation}

Ideally, the edge of the computational region should coincide with the front boundary. However, strong nonlinear variations of field $e$ or current $i$ at the front prohibit the usage of any standard interpolations for the boundary (10) since this provides reasonable accuracy only for smooth solutions. A 1D problem can replace the Neumann boundary condition with a conservation integral (9) [3], but this is not feasible for a multidimensional case. Fortunately, we can slightly extend the computational domain so that a few grid points stay in a zero field region. The algorithm iterates to choose $x_{s}\left(\tau^{n+1}\right), y_{s}\left({ }^{n+1}\right)$ in such a way that

$$
\begin{aligned}
e_{M, k}^{n+1} & =\varepsilon_{1}>0, \quad k=0, \ldots, K \\
e_{m, K}^{n+1} & =\varepsilon_{1}>0, \quad m=0, \ldots, M
\end{aligned}
$$

to enforce the zero field and

$$
\begin{array}{ll}
e_{M-1, k}^{n+1} \leq \varepsilon_{2}, & k=0, \ldots, K \\
e_{m, K-1}^{n+1} \leq \varepsilon_{2}, & m=0, \ldots, M
\end{array}
$$

to ensure negligible flux at the boundary. For a stable algorithm, $\varepsilon_{1}=10^{-300}$ was used, and sufficient accuracy was achieved with $\varepsilon_{2}=10^{-100}$.

Careful examination of advection operators reveals that the Courant number is usually greater than one, especially for initial stages of a pulse event when $x_{s}, y_{s}$ are small. This is mainly due to large multiples $u / \Delta u \sim M$ and $v / \Delta v \sim K$ in the discretization of $A$. The Courant-Friedrich-Levy condition [7] is not satisfied, and explicit methods are unstable. One possible way to overcome the problem is to move toward implicit methods. A standard upwind/downwind first-order scheme would introduce a large artificial diffusion at the front and is therefore completely unsuitable since the existence of the front is due to very sharp changes in diffusion fluxes themselves. Hence, high-order advection schemes with limiters have to be applied. Unfortunately, the only limiter that provides stable solution for a large Courant number is the "minmod" one [8]. However, tests suggest that this limiter provides only a small improvement in comparison to an upwind/downwind scheme and its usage is not desirable.

To reduce the Courant number, the motion of the boundaries was split into additional $h$ fractional steps. For each substep $g=0, \cdots, h-1$, a 1D convective problem was solved, e.g.,

$$
U\left(p^{n+(g+1) / h}-p^{n+g / h}\right)=L A_{u} p^{n+g / h}
$$

with a small displacement $\left(x_{s}^{n+(g+1) / h}-x_{s}^{n+g / h}\right)$ and the same explicit convection operator $A_{u}$. The number of displacements in (22) was chosen to keep the Courant number below unity.

A choice for limiter $L$ was studied in detail. A flux transport corrected SHASTA method was implemented together with "minmod," van Leer, Sweby, and "SuperBee" limiters [7], [8]. It was found that the smallest numerical dissipation was given by the "SuperBee" limiter, which was used for further tests.

\section{E. Pulse Event}

A very high gradient of the electric field originates during the impulse of current. It spreads very quickly in all directions. Any attempt to use directional splitting (17) and (18), or even the symmetrical form, results in unrealistically fast motion of the front. The problem can be resolved by separating a pulse into subpulses with a smaller current input during individual subpulses (similar to what was done for convection operator $A$ ). The number of subpulses in the first time step of $\sim 10 M K$ was found to give good accuracy, but this makes the algorithm slow. Alternatively, if Jacobi iterations are applied to the pulse event 


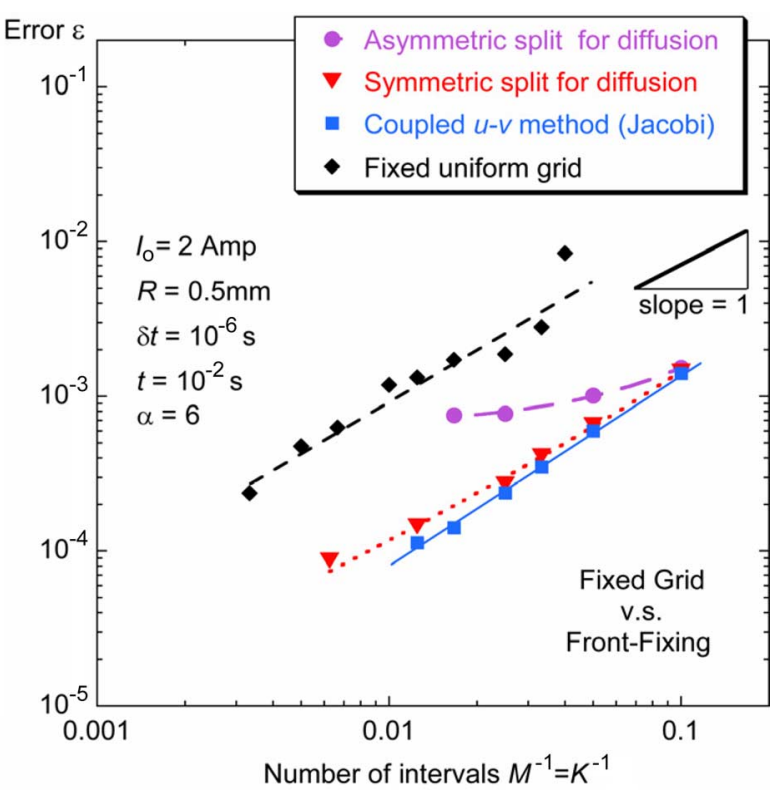

Fig. 3. Numerical prediction of electric field $E_{z}(x, y)$ for a wire with $I_{0}=$ $2 \mathrm{~A}, R=0.5 \mathrm{~mm}$, and $\alpha=6$ after $t=10 \mathrm{~ms}$ : the mesh size effects. Errors in predictions of an electric field (small time step $1 \mu \mathrm{s}$ ) reveals an approximately first order of accuracy $O\left(M^{-1}\right)$.

only (a single time step), no subpulses are required, and further calculation can be carried out with directional splitting.

\section{RESUlTS AND DisCUSSION}

Predictions from fixed grid calculations and the front-fixing method are summarized in Fig. 3, where the variations of errors with an increasing number of space intervals $M=K$ are plotted. The error $\varepsilon$ is taken in a continuous $\mathbf{C}$ norm

$$
\varepsilon=\max _{m, k=0}^{M, K}\left|e_{m, k}^{\text {analytical }}-e_{m, k}^{\text {model }}\right| \text {. }
$$

A slope of the $M^{-1}-\varepsilon$ curve in Log-Log coordinates indicates only the first-order space approximation. This is true for both fixed-grid and front-fixing approaches and is not affected by diffusion split introduced in (15)-(18). The asymmetric technique has a large directional bias; see Fig. 2(a). The bias cannot be eliminated by mesh refinement, and errors start to saturate at relatively high level. A symmetric version of the diffusion split has almost the same accuracy as the Jacobi method, but requires significantly less computational effort. In fact, the symmetric split also has a directional bias that is an inherent feature of any split technique [7]; it is just moved to the $x=y$ plane; see Fig. 2(b). Even the totally symmetric Jacobi method (19) has a slight bias around the $x=y$ plane [Fig. 2(c)], although it is hardly noticeable. The low spatial accuracy in the continuous norm is a result of the sharp front with infinite derivatives in the solution. Since the errors concentrate around the front (see Fig. 3), the convergence in any integral norm, e.g., $\boldsymbol{L}_{1}$, would be better. On the other hand, for the strongly coupled problems, the convergence in the $\mathbf{C}$ norm is of main importance.
Errors due to time discretization were studied by varying $\delta t$ between 0.05 and $1 \mathrm{~ms}$ for a large number of space intervals $M=K=200$. A combination of explicit advection with fully implicit diffusion provides a first-order approximation in time $O(\delta t)$ as expected. Such choice is dictated by high field (current) gradients during the pulse event. When the pulse disperses slightly, it is possible to move toward the semi-implicit method with $O\left(\delta t^{2}\right)$ [3] by applying Crank-Nicolson approximations for convection and diffusion operators. The method can be improved further by the Lax-Wendroff explicit approach with limiters and by the alternating direction implicit (ADI) scheme [7]. The latter should make the method approximately symmetric with reduced directional bias. (A small asymmetry will still remain due to the inherent property of the ADI method, where an implicit scheme is applied in one direction while an explicit one in the other.)

\section{CONCLUSION}

Generally, there are several advantages of using a front-fixing method for modeling of impulse phenomena in HTS. In particular, high accuracy can be obtained with a small number of grid points and large time increments. However, the following properties of the solution need to be noted and handled carefully.

1) Complex boundary conditions have to be implemented by considering the conservation laws.

2) A careful choice of a limiter for advection problems associated with the mesh motion needs to be made.

3) Fully implicit schemes may be needed for pulse events, thus limiting temporal accuracy. It is suggested to switch toward semi-implicit methods after the pulse disperses.

\section{REFERENCES}

[1] K. Berger, J. LeVeque, D. Netter, B. Douine, and A. Rezzoug, "AC transport losses calculation in a Bi-2223 current lead using thermal coupling with an analytical formula," IEEE Trans. Appl. Supercond., vol. 15 , no. 2 , pt. 2 , pp. 1508-1511, Jun. 2005.

[2] J. Crank, Free and Moving Boundary Problems. Oxford, U.K.: Clarendon, 1984.

[3] I. O. Golosnoy and J. K. Sykulski, "Evaluation of the front-fixing method capabilities for numerical modelling of field diffusion in high-temperature superconducting tapes," IET Sci. Meas. Techn., vol. 2, no. 6, pp. 418-426, 2008.

[4] J. Rhyner, "Magnetic properties and AC-losses of superconductors with power law current-voltage characteristics," Phys. C, vol. 212, p. 292, 1993.

[5] J. K. Sykulski, R. L. Stoll, and A. E. Mahdi, "Modelling HTc superconductors for AC power loss estimation," IEEE Trans. Magn., vol. 33, no. 2, pt. 2, pp. 1568-1571, Mar. 1997.

[6] G. J. Pert, "A class of similar solutions of the non-linear diffusion equation,” J. Phys. A, Math. Gen., vol. 10, pp. 583-593, 1977.

[7] J. W. Thomas, Numerical Partial Differential Equations: Conservation Laws and Elliptic Equations. New York: Springer, 1999.

[8] R. J. LeVeque, Finite Volume Methods for Hyperbolic Problems. Cambridge, U.K.: Cambridge Univ. Press, 2002.

[9] T. C. Illingworth and I. O. Golosnoy, "Numerical solutions of diffusion-controlled moving boundary problems which conserve solute," $J$. Comput. Phys., vol. 209, pp. 207-225, 2005.

[10] T. C. Illingworth, I. O. Golosnoy, V. Gergely, and T. W. Clyne, "Numerical modelling of transient liquid phase bonding and other diffusion controlled phase changes," J. Mater. Sci., vol. 40, pp. 2505-2511, 2005. 